\title{
Ichthyofaunal Biodiversity of Kharakuva Fish Market, Veraval, Gujarat, India
}

\author{
Arti Joshi ${ }^{1 *}$, Ejaz A. Rahim Parmar' ${ }^{2}$, G. S. Temkar ${ }^{3}$, A. Y. Desai ${ }^{4}$ and A. J. Bhatt ${ }^{5}$ \\ ${ }^{182}$ Veraval research centre of ICAR- Central Institute of Fisheries Technology, Matsya Bhavan, Bhidia, Veraval, \\ Gujarat (362 269), India \\ ${ }^{3,485}$ College of Fisheries Science, JAU, Veraval, Gujarat (362 265), India
}

\section{Corresponding Author}

Arti Joshi

e-mail: rtjoshi0@gmail.com

\author{
Article History \\ Article ID: AR1902 \\ Received in $18^{\text {th }}$ September, 2018 \\ Received in revised form $07^{\text {th }}$ October, 2018 \\ Accepted in final form $11^{\text {th }}$ October, 2018
}

\begin{abstract}
The coastline of Gujarat is $1,600 \mathrm{~km}$ long and salt marshes, sand-belts and gravel patches mark the topography. During the present study, total 94 finfish and 26 shellfish species belonging to 62 families and 18 orders were identified fromthe Kharakuva Fish market of Veraval Taluka from Gir-Somanth district of Gujarat, India. Data were collected with the help of local skilled fishermen and fish farmers from different locations of the different area forAugust, 2017 to March, 2018 on the ichthyofaunal diversity of Veraval coast, Gujarat. Identification of fishes was based on fresh or preserved specimens. They were identified by using standard taxonomic keys. The collected fish were identified up to species level. During the present study there were 94 finfish species under 14 order and 52 families, whereas 26 shellfish species belonging to 4 orders and 10 families were reported. Among the finfishes order Perciformes while in case of shellfishes order Decapoda represents abundance in landing. In case of family wise contribution Carangidae contributes a greater number of finfish species and family Penaeidae contributes a greater number of shellfish species. The price structure reported that shellfish fetches good value than that of finfish. Among finfish Protonibea diacanthus (Jew fish) and among the shellfish lobsters viz., Penulirus homarus and $P$. ornatus (Spiny lobster) fetches the highest price.
\end{abstract}

Keywords: Ichthyofaunal, fish diversity, kharakuva fish market, veraval

\section{Introduction}

Biodiversity is the variation in the genetics and life forms of populations, species, communities and ecosystem. It affects the capacity of living system to respond to changes in the environment and is essential for providing goods and services from ecosystems (Rahbek and Colwell, 2011). The protection and conservation of biodiversity is important for the sustainability of marine natural resources. The studies were evidence that the fisheries which were exploit multispecies diversity reports the stable catches with less harm to the marine resources than that of the single targeted species fisheries (Dulvy et al., 2000; Hilborn et al., 2003).

Ichthyofaunal diversity refers to variety of fish species. India is one of the 17 mega biodiversity countries of the world, with only $2.5 \%$ of the land area, India accounts for $7.8 \%$ of the recorded species of the world (Venkataraman, 2006). The variety of fishes includes the variation among the genotype and so species diversity within species population (Johnson et al., 2016). The marine fishes were varies in their size from smallest Goby fish (up to $8 \mathrm{~mm}$ ) to that of Rhincodon typus, Whale Shark (may be reach up to $12 \mathrm{~m}$ ). The greater diversity was observed in the sea than that of land, so the exploitation rate was also more for sea resources (Geoffrey et al., 2007). For time immoral fishes were used by the human beings for the various purposes. The major utilization of fishes is as food resource. The large population throughout the world is affected by hunger and malnutrition, for them fish will be one of the good resource to fulfill their demand. Presently fish resource becomes as the major protein provider to the world's population, reports were also stated that, the developing countries were more depends on fish as source of food (Bene et al., 2007). Apart from food, marine fisheries also contribute for sport and ornamental business, which explains marine fish resource was always extremely valuable economically. In the recent past commercialization was increased very rapidly and that impacted as over exploitation of certain species to fulfillment of social and economic needs of fisher's population (Walters and Pearse, 1996). During last five-six decades due to large scale harvesting of some valuable and important fish species, resources goes on the verge of extinction. So for sustainable future of marine resources it is need to application of better policies and management strategies supported by good scientific knowledge (Kabat et al., 2012). 
Throughout the world as per the Fishbase website report there were about 28,900 living species of fish have been recorded (Levque et al., 2008). While,further William et al. (2010) reported there were total of 31,362 distinct fish species available globally. According to the IUCN (2008) there were about 2,544 species of fishes reported as threatened fishes on earth i.e. Red list species. The earlier report along Indian waters by Talwar (1991) stated that, there were a total of 2,546 fish species were recorded from Indian waters, under 254 families and 40 orders. Further Kar et al. (2003) stated there werea total 2,500 species of fishes recorded in India, amongst them 930 live in freshwater and 1,570 are marine. Another report in 2011 specified in India, total 2,358 number of finfishes were recorded and among them 877 species are fresh water, while 113 species are brackish water and 2,358 are marine species (Ayyappan et al., 2011).

The present study location is situated in Gujarat state of India. The coastline of Gujarat is 1,600 km long,with salt marshes, sand-belts and gravel patches mark the topography. Marine ecosystems are extraordinarily diverse in all aspects; it varied from genetic to taxonomic to ecological level. About $75 \%$ of the marine fish in Gujarat are landed at 10 major centres. Veraval is the most important one, with landings of over 0.17 $\mathrm{mt}$, which is $27 \%$ of the total landings of the state (CMFRI, 2017). Other major centers include Porbandar, Mangrol, Jafrabad and Okha. Most of the fresh fishes are available at the Kharakuva, Divadandi jetty, Jaleshwar landing centers and other most of local fresh market.In Veraval, fish comes from all nearby small and big harbors and landing centers due to the demand of processing plant and export companies. That causes the market price of fish is also high. The Veraval Kharakuva fresh fish market have total 190-200 shops and the number of fish merchant (Bepari) is around 220-230. Amongst them 200 fish merchants were having the license. In the Kharakuva fresh fish market chilled fish were supply in the domestic market, it means to all over India. The aim of this study was to investigate the current ichthyofaunal diversity and provide the first systemic account of fishes reported along the Veraval coast, Gujarat.

\section{Materials and Methods}

\subsection{Data collection}

The present survey study was conducted along the coastal waters of Veraval $\left(21^{\circ} 35^{\prime} \mathrm{N}, 69^{\circ} 36^{\prime} \mathrm{E}\right)$, India which is situated along the western coast of Gujarat, India. The data was collected with the help of local fishermen landings and by visiting the Kharakuva fish market, which is located in Veraval Taluka of Gir-Somnath district from Gujarat state of India. Samplings was done at fortnightly intervals. Sampling was carried out during the period from August, 2017 to March, 2018. Samples were collected from different fishing gears viz., gill net, hook and line, trawl net, purse seine from different mesh size. The collected fishes were initially treated with $8 \%$ formalin for 48 hours during identification and finally preserved in 5\% formalin for long term storage.

\subsection{Identification of fishes}

Identification of fishes was based on fresh or preserved specimens. They were identified by using standard taxonomic keys viz. Day (1878), Talwar and Jhingran (1991), Anonymous $(2018 ; a, b, c)$. The collected fish were identified up to species level.

\section{Results and Discussion}

As per the Central Marine Fisheries Research Institute report Gujarat is top fish producing state in the India, this highest landing rank was maintained by Gujarat since from last 4 years constantly (CMFRI, 2017). They reported that in the year of 2016 landings of Gujarat state was $0.77 \mathrm{mt}$, which contributed around $21.32 \%$ to the total fish landings of India. While in the Gujarat state the Gir-Somnath district contributes maximum landings i.e. 0.34 lakh tones and approx. $44 \%$ to total Gujarat landings and from this district the present study area i.e. Veraval coast stood first in the landings. As the human population is ever increasing, it means that less fish will be available per caputevery year. The handling, processing, and marketing of fish products are essential complementary functions of all food production systems. The marketability of fish products is an important constraint in the development of fisheries. Moreover, processing and marketing activities provide the greatest opportunities for employment within the fisheries industry.During the present study, there were total 94 finfish and 26 shellfish species belonging to 62 families and 18 orders were identified fromthe Kharakuva Fish market of Veraval Taluka from Gir-Somanth district of Gujarat (Table 1 and Table 2).

There were total 52 families of finfish species were reported during the present study. Amongst them Carangidae contributes a greater number of finfish species (9 spp.), which was followed by Scombridae (7 spp.), Sciaenidae (6 spp.), Synodontidae (5 spp.), Clupeidae (4 spp.), Polynemidae, Carcharhinidae, Myliobatidae, Ariidae (3 spp. each), Stromateidae, Istiophoridae, Serranidae, Haemulidae, Sphyraenidae, Trichiuridae,Muraenesocidae,Hemiramphidae ( 2 spp. each) and remaining 35 families reported one species each of observed fin fish diversity (Figure 1).

The order wise observations of finfish representPerciformes was the most abundant with $54.26 \%$, which was further followed by Clupeiformes (8.51\%), Carcharhiniformes (5.32\%), Aulopiformes (5.32\%), Pleuronectiformes (4.26\%), Myliobatiformes (4.26\%), Beloniformes (4.26\%) and Tetraodontiformes (3.19\%; Figure 2). The Kharakuvalocal fish market price study stated that the fish price varies between ₹ 20-1,500/-. The highest price fetched fishes in the market were Jew fish (Ghol), Silver pomfret, Chinese pomfret, Indian scad, Chinese herring, Silver conger eel, Sharks, Ribbon fishes etc. (Table 1; Figure 5). The highly valued fishes mostly consumed fresh and remaining catch goes to the processing 


\begin{tabular}{|c|c|c|c|c|c|}
\hline Order & Family & Scientific name & Trade name & Local name & $\begin{array}{l}\text { Price } \\
\left(₹ \mathrm{~kg}^{-1}\right)\end{array}$ \\
\hline \multirow[t]{38}{*}{ Perciformes } & Stromateidae & Pampus argenteus & Silver pomfret & Paplet, Vichudo & $500-1000$ \\
\hline & & P. chinensis & Chinese pomfret & Gadiyo, Kafari & $500-1000$ \\
\hline & Carangidae & Formio niger & Black pomfret & Halvo, Ardiyo & $300-320$ \\
\hline & & Megalaspis cordyla & Horse mackerel & Bangda & $80-90$ \\
\hline & & Atropus atropus & Kuwesh travelly & Nariyela & $150-160$ \\
\hline & & Caranx sexfasciatus & Dusky travelly & Nariyela & $150-160$ \\
\hline & & Decapterus russelli & Russell's scad & Khota bangda & $50-60$ \\
\hline & & Carangoides malabaricus & Malabar travelly & Khadva & $120-140$ \\
\hline & & C. coeruleopinnatus & Coastal travelly & Khadva & $130-140$ \\
\hline & & Decapterus macrosoma & Shortfin scad & Dolly & $30-35$ \\
\hline & & Elagatis bipinnulata & Rainbow runner & Maru / Popat & $60-70$ \\
\hline & Scombridae & Rastrelliger kanagurtra & Indian mackerel & Malbari bangda & $85-90$ \\
\hline & & Euthynnus affinis & Little tuna & Gedra & $70-80$ \\
\hline & & Auxis thasard & Frigate tuna & Gedri & $50-60$ \\
\hline & & Katsuwonus pelamis & Skipjack tuna & Gedra/ Potla & $70-80$ \\
\hline & & Thunnus albacarus & Yellow fin tuna & Vir gedra & $110-120$ \\
\hline & & T. toggol & Long tail tuna & Serva & $140-150$ \\
\hline & & T. obesus & Big eye tuna & Serva & $140-150$ \\
\hline & Polynemidae & Polynemus tetradactylus & Four finger thread fin & Dara / Bava & $300-350$ \\
\hline & & P. indicus & Indian thread fin & Ravas & $300-350$ \\
\hline & & P. heptadactylus & Seven finger thread fin & Sheri & $250-280$ \\
\hline & Sciaenidae & Johnius dussumieri & Bearded croaker & Mathara dhoma & $55-60$ \\
\hline & & Otolithes cuvieri & Lesser tooth croaker & Silver dhoma & $65-70$ \\
\hline & & O. ruber & Tiger tooth croaker & $\begin{array}{l}\text { Mosambi dhoma/TT } \\
\text { dhoma }\end{array}$ & $130-140$ \\
\hline & & O. biauritus & Bronze croaker & Goyni/Koth & $300-350$ \\
\hline & & Nibea maculate & Blotched croaker & Babar & $70-75$ \\
\hline & & Protonibea diacanthus & Jew fish & Ghol & $900-1500$ \\
\hline & Istiophoridae & Istiophorus platypterus & Sail fish & Ghodo & $95-100$ \\
\hline & & Makaira indica & Black marlin & Ghodo & $85-90$ \\
\hline & Serranidae & Epinephelus diacanthus & Six barred reef cod & Lal vekhlu & $180-200$ \\
\hline & & E. tauvina & Grey reefcod & Vekhlu & $240-270$ \\
\hline & Haemulidae & Pomadasys argenteus & Silver grunt & Ghurko & $170-180$ \\
\hline & & Diagramma pictum & Painted sweet lip & Aandar & $160-170$ \\
\hline & Xiphiidae & Xiphias gladius & Sword fish & Surajmukhi & $80-90$ \\
\hline & Sphyraenidae & Sphyraena obtusata & Obtuse barrakuda & Jeera & $90-100$ \\
\hline & & S. jello & Gaint sea pike, barrakuda & Jeero, Kunga & $100-110$ \\
\hline & Lethrinidae & Lethrinus ornatus & Ornate emperor bream & Dhamil & $220-250$ \\
\hline & Lactariidae & Lactarius lactarius & White fish & Khitli & $130-140$ \\
\hline
\end{tabular}




\begin{tabular}{|c|c|c|c|c|c|}
\hline Order & Family & Scientific name & Trade name & Local name & $\begin{array}{l}\text { Price } \\
\left(₹ \mathrm{~kg}^{-1}\right)\end{array}$ \\
\hline \multirow[t]{13}{*}{ Perciformes } & Rachycentridae & Rachycetron canadum & Cobia & Sakro & $270-300$ \\
\hline & Menidae & Mene maculata & Moon fish & Chand fish & $25-30$ \\
\hline & Coryphaenidae & Coryphaena hippurus & Dolphin fish & Apnus & $150-170$ \\
\hline & Echeneidae & Remora remora & Sucker fish & Meghar & $20-25$ \\
\hline & Terapontidae & Terapon jarbua & Jarbua terapon & Khaturo / Hajam & $25-30$ \\
\hline & Gerreidae & Gerres filamentosus & Whipfin majorra & Todyu & $70-80$ \\
\hline & Lutjanidae & Lutjanus johni & Golden snapper & Raja, Gulaliyo & $240-250$ \\
\hline & Nemipteridae & Nemipterus japonicas & Thread fin breams & Rani fish / Lal machla & $80-90$ \\
\hline & Priacanthidae & Priacanthus hamrur & Bull's eye & Dola, Dorara & $30-50$ \\
\hline & Mullidae & Upeneus sulphureus & Yellow goat fish & Khota lal machla & $30-40$ \\
\hline & Drepaneidae & Drepane punctala & Spotted sickle fish & Dafniyo & $80-100$ \\
\hline & Trichiuridae & Lepturacanthus savala & Silver ribbon fish & Bagga, Silver bagga & $170-180$ \\
\hline & & Trichiurus lepturus & Grey head hair tail & Bagga, Black bagga & $150-170$ \\
\hline \multirow{5}{*}{$\begin{array}{l}\text { Carcharhini- } \\
\text { formes }\end{array}$} & Carcharhinidae & Carcharias melnopteruts & Black shark & Patari & $160-170$ \\
\hline & & C. limbatus & Black tip shark & Magra & $100-130$ \\
\hline & & Scoliodon laticaudus & Spadenose shark & Sandho & $80-90$ \\
\hline & Sphyrnidae & Sphyrna lewini & Hammer head shark & Nathiyo & $110-120$ \\
\hline & Triakidae & Mustelus mosis & Arabian smooth hound & Bokha & $70-80$ \\
\hline \multirow[t]{5}{*}{$\begin{array}{l}\text { Aulopi- } \\
\text { formes }\end{array}$} & Synodontidae & Saurida tumbil & Grater lizard fish & $\begin{array}{l}\text { Bhungar / Chor } \\
\text { bumla }\end{array}$ & $35-40$ \\
\hline & & S. undosquami & Brush tooth lizard fish & $\begin{array}{l}\text { Bhungar / Chor } \\
\text { bumla }\end{array}$ & $30-35$ \\
\hline & & Saurida longimanus & Long fin lizard fish & Pencil bhungar & $35-40$ \\
\hline & & Trachinocephalus myops & Bluntnose lizard fish & Patta & $25-30$ \\
\hline & & Harpodon nehereus & Bombay duck & Bumbla / Danntaniya & $30-40$ \\
\hline \multirow{4}{*}{$\begin{array}{l}\text { Pleuronecti- } \\
\text { formes }\end{array}$} & Paralichthyidae & Pseudorhombus arsius & Large toothed flounder & Khetar, Datari & $60-80$ \\
\hline & Psettodidae & Psettodes erumei & Indian halibut & Hariyo & $100-110$ \\
\hline & Cynoglossidae & Cynoglossus lingua & Long tongue sole & Moti jibh & $75-80$ \\
\hline & Soleidae & Zebrias quagga & Zebra sole & Jibh & $30-40$ \\
\hline \multirow{4}{*}{$\begin{array}{l}\text { Myliobati- } \\
\text { formes }\end{array}$} & Myliobatidae & Mobula diabolus & Devil ray & Timri & $80-90$ \\
\hline & & Rhinoptera javanica & Cow ray & Bur & $100-105$ \\
\hline & & Aetomylaeus nichofii & Neiuhof's eagle ray & Karaj & $60-70$ \\
\hline & Dasyatidae & Dasyatis zugei & Pale - edged sting ray & Varara & $90-100$ \\
\hline \multirow{8}{*}{$\begin{array}{l}\text { Clupe- } \\
\text { iformes }\end{array}$} & Clupeidae & Tenualosa ilisha & Indian scad & Chaksi & $800-900$ \\
\hline & & T. toil & Chinese herring & Palvi, Palvo & $500-600$ \\
\hline & & Sardinella longiceps & Oil sardine & Aed & $30-40$ \\
\hline & & Opisthopterus tardoore & Tardoore & Khoti katti & $25-30$ \\
\hline & Dussumieriidae & Dussumeria acuta & Rainbow sardine & Telas katti & $20-25$ \\
\hline & Engraulidae & Colia dussumieri & Golden anchovy & Mendli & $10-15$ \\
\hline & Chirocentridae & Chirocentrus dorab & Silver bar & Dai & $80-100$ \\
\hline & Pristigasteridae & Ilisha megaloptera & Big eye ilisha & Katti, Dorari katti & $30-35$ \\
\hline
\end{tabular}




\begin{tabular}{|c|c|c|c|c|c|}
\hline Order & Family & Scientific Name & Trade name & Local name & $\begin{array}{l}\text { Price } \\
\left(₹ \mathrm{~kg}^{-1}\right)\end{array}$ \\
\hline \multirow[t]{2}{*}{ Rajiformes } & $\begin{array}{l}\text { Rhynchobati- } \\
\text { dae }\end{array}$ & Rhynchobatus djiddensis & $\begin{array}{l}\text { Guitar fish, white spotted } \\
\text { shovel nose ray }\end{array}$ & Ghose, Bhuttar & $150-200$ \\
\hline & Rhinobatidae & Rhynobatos granulatus & $\begin{array}{l}\text { Granulated shovel-nose } \\
\text { ray }\end{array}$ & Ghose, Bhuttar & $150-200$ \\
\hline \multirow[t]{2}{*}{$\begin{array}{l}\text { Anguillifor- } \\
\text { mes }\end{array}$} & $\begin{array}{l}\text { Muraenesoci- } \\
\text { dae }\end{array}$ & Congresox talabonoides & Indian pike conger & Vaam & $230-250$ \\
\hline & & Muraenesox cinereus & Silver conger eel & Vaam & $500-550$ \\
\hline \multirow[t]{3}{*}{ Siluriformes } & Ariidae & Arius thalassinus & Giant catfish & Khago, Khagi & $150-160$ \\
\hline & & A. dussumieri & Dussumieri's catfish & Khago, Khagi & $60-70$ \\
\hline & & Osteogeniosus militarius & Soldier catfish & Goji khagi & $200-250$ \\
\hline \multirow{4}{*}{$\begin{array}{l}\text { Beloni- } \\
\text { formes }\end{array}$} & Exocoetidae & Cypselurus oligolepis & Large scaled flying fish & Pankhiya jira & $50-60$ \\
\hline & $\begin{array}{l}\text { Hemiramphi- } \\
\text { dae }\end{array}$ & Hemiramphus far & Black barred half beak & Chanchiya jira, kunga & $40-50$ \\
\hline & & H. lukei & Lutke's half beak & Tori & $70-75$ \\
\hline & Belonidae & Strongylura stongylura & Full beak gar fish & Chanchiya jira & $75-80$ \\
\hline Pristiformes & Pristidae & Pristis microdon & Small toothed saw fish & $\begin{array}{l}\text { Churiyu, Surya mag- } \\
\text { ru }\end{array}$ & $45-50$ \\
\hline $\begin{array}{l}\text { Scorpaeni- } \\
\text { formes }\end{array}$ & Platycephalidae & Platycephalus indicus & Indian flathead & Gokhin & $20-25$ \\
\hline $\begin{array}{l}\text { Mugili- } \\
\text { formes }\end{array}$ & Mugilidae & Mugil cephalus & Grey mullet & Boy, Gandhiyo & $50-60$ \\
\hline \multirow{3}{*}{$\begin{array}{l}\text { Tetraodonti- } \\
\text { formes }\end{array}$} & Triacanthidae & Pseudotriacanthus strigilifer & Long-spined tripod fish & Helicopter & $15-20$ \\
\hline & Monacanthidae & Aluterus monoceros & Unicorn letherjacket & Don & $110-120$ \\
\hline & Balistidae & Abalistes stellaris & Starry triggerfish & Gagira & $25-30$ \\
\hline
\end{tabular}

1 US\$=INR 64.47 (avg. of August 2017 to March 2018)

plants, from where after value addition and freezing those were exported to the other countries.

Among the 26 species of shellfish there were total 10 families and 4 orders were observed. The order Decapoda was most abundant comprising with total 20 species $(76.92 \%)$ of crustaceans (Figure 3 and 4). Among the order Decapoda, family Penaeidae consist of 8 species which was followed by Palinuridae, Portunidae, Sepiidae by contributing each of 4 shellfish species (Table 2). The comparison between the Kharakuva market price structures it was observed that crustaceans shows higher market value than that of finfishes. This is mainly because of their feeding preference, taste and higher demand among the consumer population. The lobsters fetch the higher amount than that of shrimps and cephalopod species among shellfishes. Crabs and non-penaeid shrimps were mostly preferred by local fish consumers (Figure 6).

The fisheries of any natural ecosystem was based relatively on large number of species and a wide range of fishing gears. Habitat degradation, invasion of exotic fishes and fishing pressure are the main causes for loss of fish biodiversity.
Study reports from Central Marine Fisheries Research Institutedescribedthat, India contributes of about 2,492 marine fishes (7.4\%) of the total world marine fish resources. While from the total reported fish diversity from India, the marine fishes constitute of $76 \%$ (2,492 spp.) which belonging to 941 ordersand 240 families. Study also reported that Andaman and Nicobar archipelago water represents the highest number of species diversity i.e. 1,431species, which was followed by the east coast of India with 1,121 species and the west coast with 1,071species (Joshi et al., 2017).

Some of the local studies reported as follows; from Karaikal waters, southeast coast of India there were total 195 species belonging to 18 orders, 87 families and 134 genera which includes Stolephorus indicus, Thryssa malabarica, T.purava, Chirocentrus dorab, Arius arius, Plotosus canius, Mugil cephalus, Hemiramphus far, Platycephalus indicus, Epinephelus tauvina, E. malabarica, Sillago sihama, Caranx sem, Scomberoidestol, Lutjanus fulviflamma, Gerres abbreviatus, Upeneus sulphureus, Drepane punctata, Terapon puta, Trichiurus lepturus, Pampus argenteus, P. chinensis, 


\begin{tabular}{|c|c|c|c|c|c|}
\hline Order & Family & Scientific Name & Trade name & Local name & Price $\left(₹ \mathrm{~kg}^{-1}\right)$ \\
\hline \multirow[t]{20}{*}{ Decapoda } & Solencoceridae & Solenocera crassicornis & Coastal mud prawn & Lal kolmi, Goiner & $70-80$ \\
\hline & \multirow[t]{7}{*}{ Penaeidae } & Parapenaeopsis stylifera & Kiddi prawn & Tiny kolmi & $110-120$ \\
\hline & & Metapenaeus affinis & Indian prawn & Medium & $250-300$ \\
\hline & & Metapenaeus kutchensis & Kutch prawn & Surajbari, Katchi jinga & $150-200$ \\
\hline & & Metapenaeus Monoceros & Speckled prawn & Kapsi & $280-300$ \\
\hline & & Penaeus indicus & Indian white prawn & White, Jumbo & $380-400$ \\
\hline & & Penaeus monodon & Tiger prawn & Tiger & $600-700$ \\
\hline & & Penaeus semisulcatus & Flower prawn & Flower & $650-700$ \\
\hline & \multirow[t]{2}{*}{ Hippolytidae } & Parapenaeopsis sculptilis & Rainbow prawn & Patta & $100-120$ \\
\hline & & Exhippolysmata ensirostris & Hunter shrimp & Dodi & $80-90$ \\
\hline & Sergestidae & Acetes indicus & Paste shrimp & Javlo & $8-10$ \\
\hline & \multirow[t]{4}{*}{ Palinuridae } & Penulirus polyphagus & Rock lobster & Titan & $800-1000$ \\
\hline & & Penulirus homarus & Spiny lobster & Titan & $900-1000$ \\
\hline & & Penulirus ornatus & Spiny lobster & Titan & $900-1000$ \\
\hline & & Thenus orientalis & Sand lobster & Kako & $280-300$ \\
\hline & Scyllaridae & Sepia pharaonis & Cuttle fish & Makul & $230-250$ \\
\hline & \multirow[t]{4}{*}{ Portunidae } & Charybdis cruciata & Cross crab & Lal karchlo & $70-80$ \\
\hline & & Scylla serrata & Mud crab & Dhebro karchlo & $50-60$ \\
\hline & & Portunus pelagicus & Blue crab & Blue karchlo & $70-80$ \\
\hline & & Portunus sanguinolentus & Three spotted crab & Tapkavaro karchlo & $80-90$ \\
\hline \multirow[t]{4}{*}{ Sepiida } & \multirow[t]{4}{*}{ Sepiidae } & Sepia aculeata & Needle cuttlefish & Dedka & $200-300$ \\
\hline & & Sepia prasadi & Cuttle fish & LB (long bone) & $200-300$ \\
\hline & & Sepiella inermis & Spineless Cuttle fish & Inda goti, Goti & $100-110$ \\
\hline & & Photo Loligo duvaucelli & Squid & Narsinga & $150-200$ \\
\hline Teuthida & Loliginidae & Doryteuthis sibogae & Needle squid & Needle & $130-140$ \\
\hline Octopoda & Octopodidae & Octopus globosus & Octopus & Salak, Sikavdo & $70-90$ \\
\hline
\end{tabular}

1 US\$=INR 64.47 (avg. of August 2017 to March 2018)

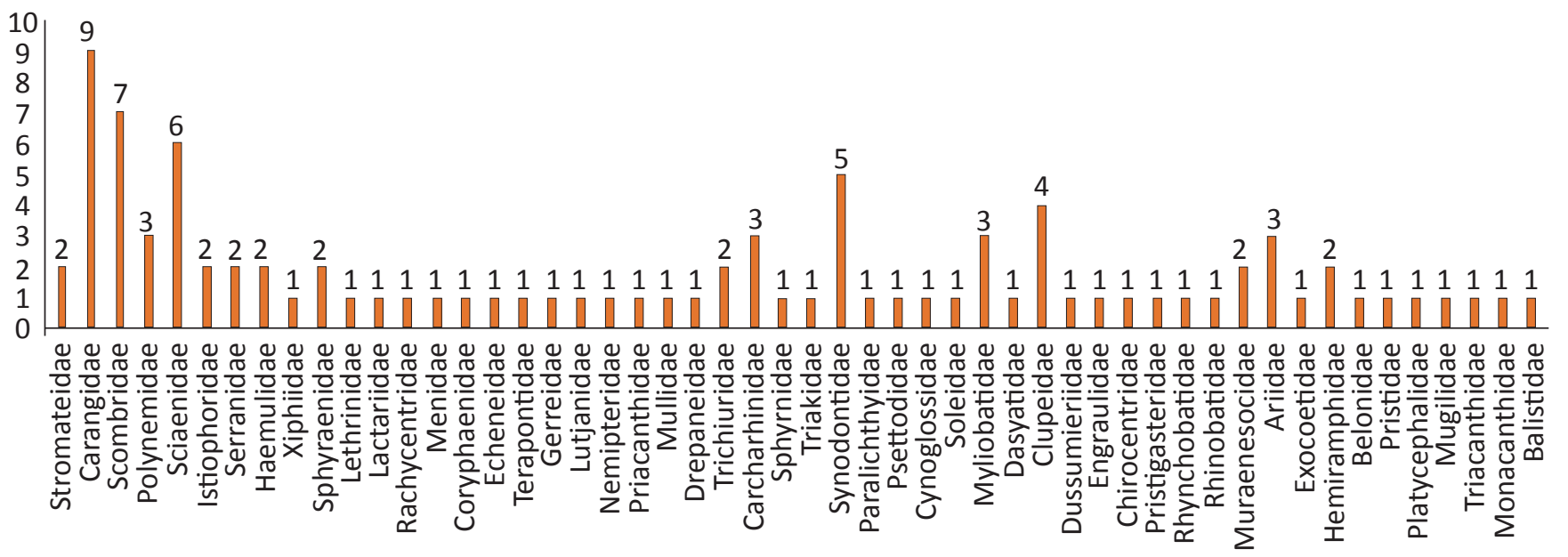

Figure 1: Diagrammatic representation of the number of species occurring in each Finfish Family

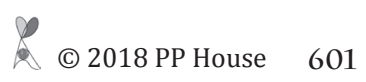




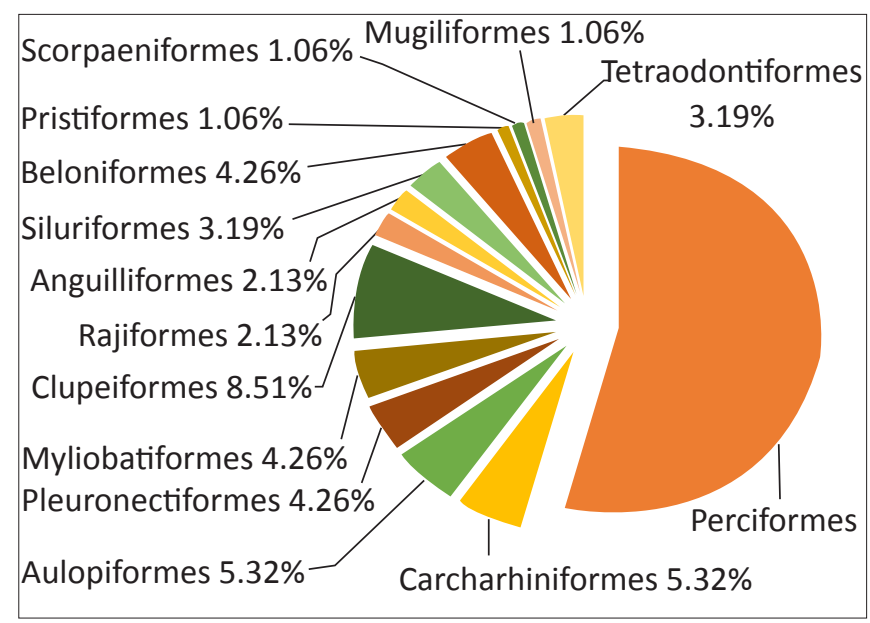

Figure 2: Diagrammatic representation of \% contribution in each Finfish Order

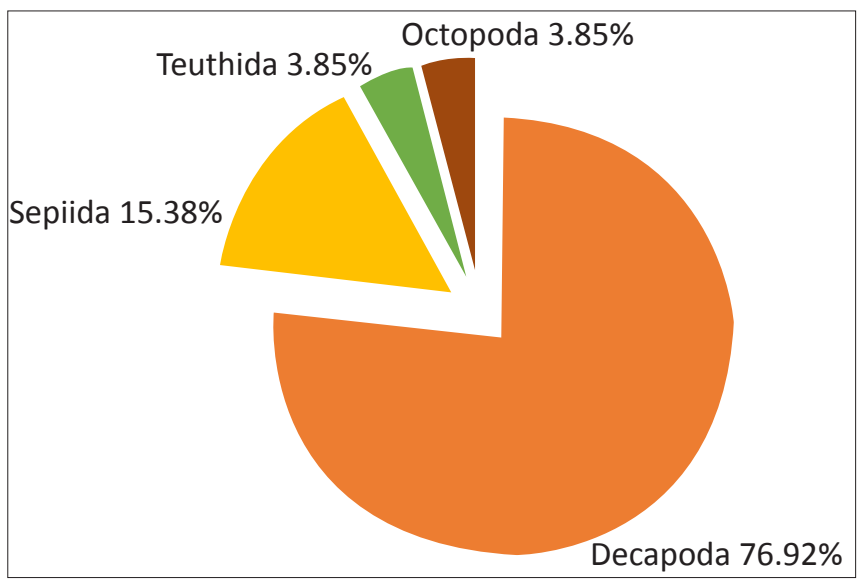

Figure 3: Order-wise distribution of Shellfish

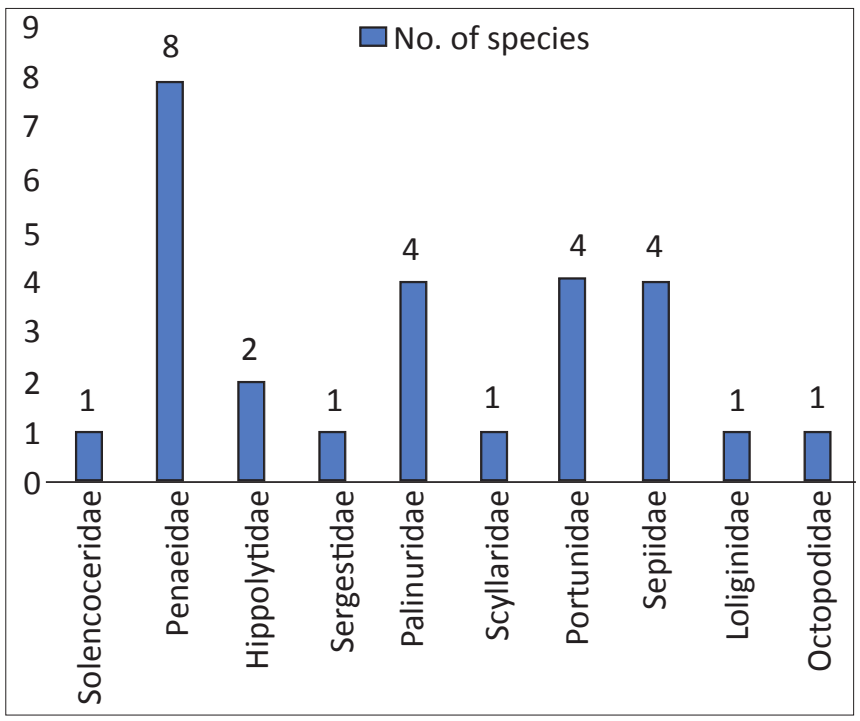

Figure 4: Family-wise distribution of Shellfish Species

Cynoglossus arel and Triacanthus biacculeatus were reported (Rajasegar and Sendhilkumar, 2009). The Ichthyofaunal diversity of Ponnani estuary, Kerala documented 112 species
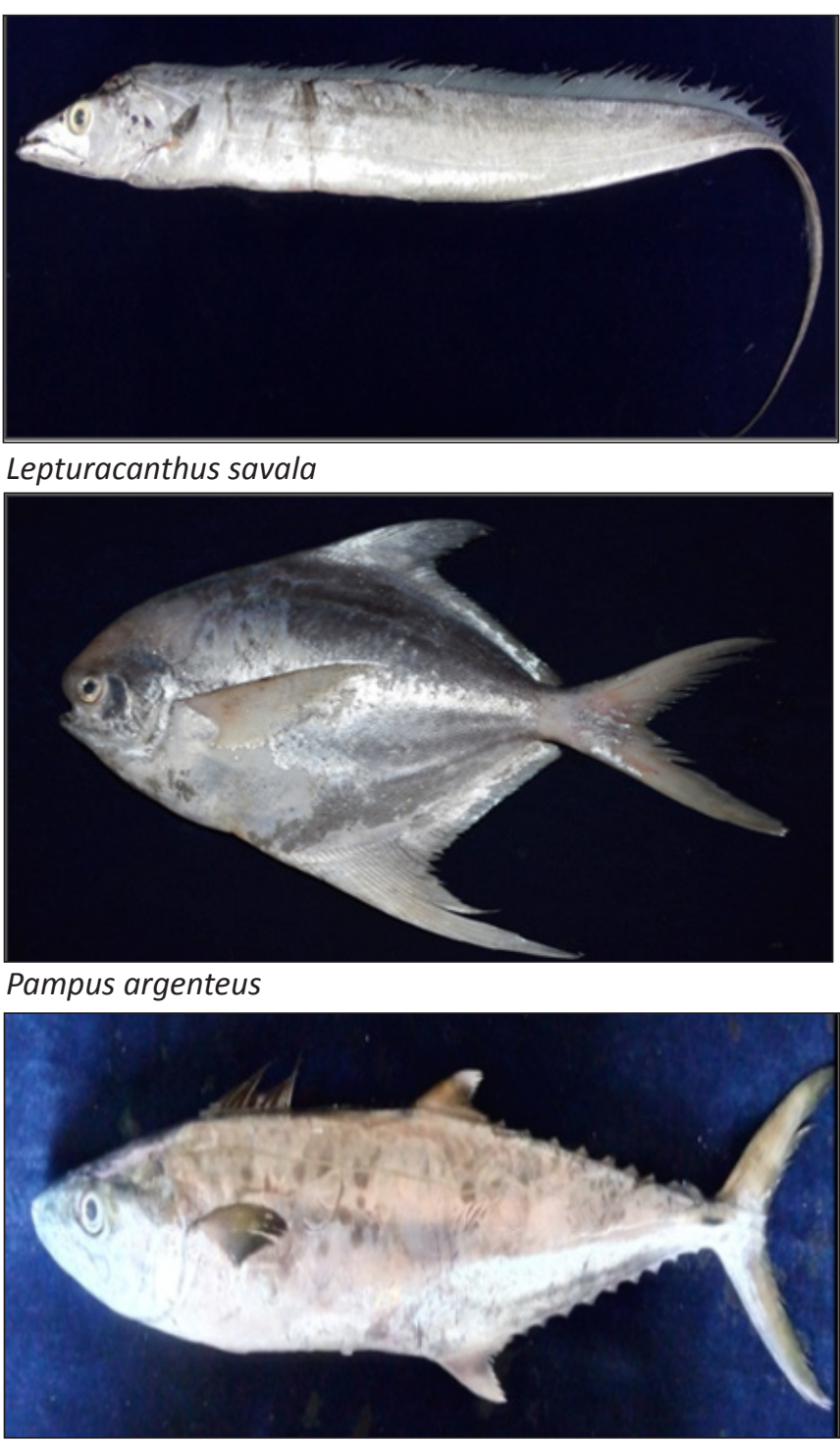

Scomberomorus guttatus

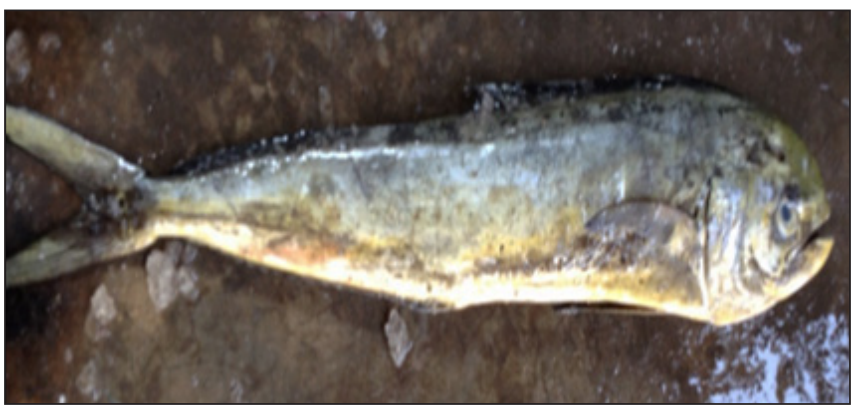

Coryphaena hippurus

Figure 5: Finfish species available at Kharakuva Fish Market (Gujarat)

belonging of 14 orders, 53 families and 80 genera (Bijukumar and Sushama, 2000), whereas from Giriyampeta estuary, Yanam (U.T. of Puducherry) 36 species were identified with 13 families which includes 30 species, the order Clupeiformes reported a greater number of fishes (Kumaran et al., 2012). 


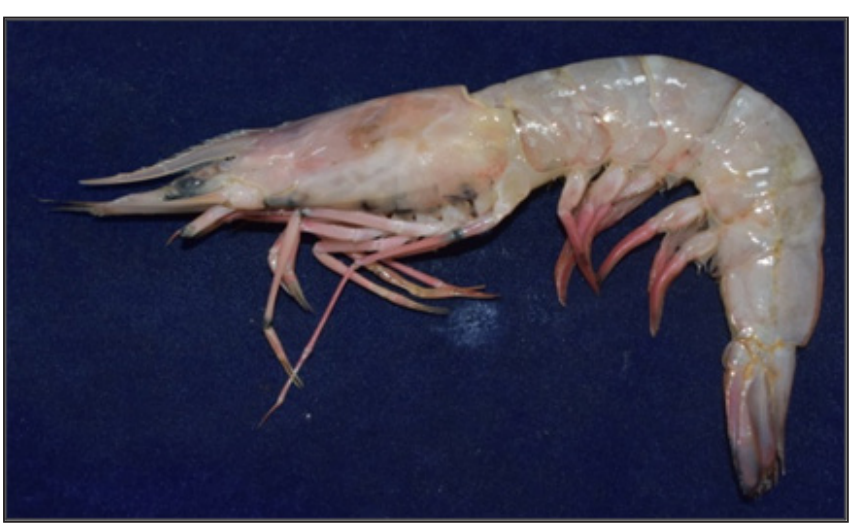

Metapenaeus affinis

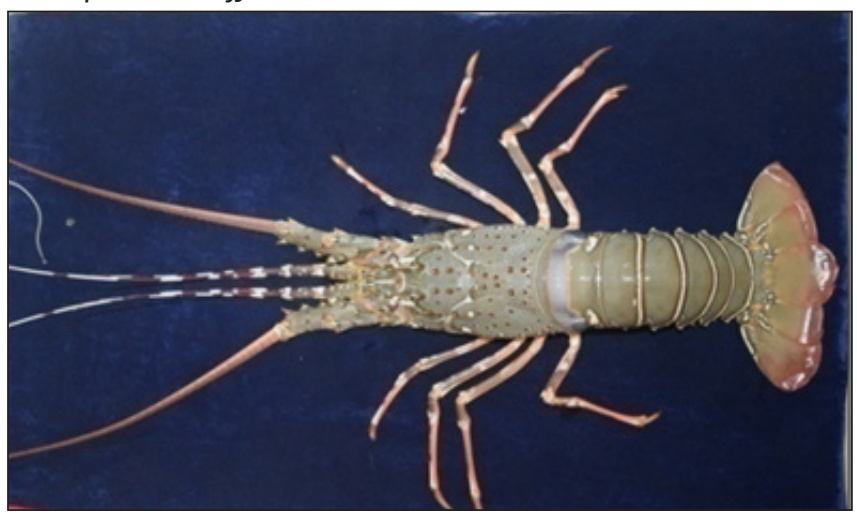

Panulirus homarus

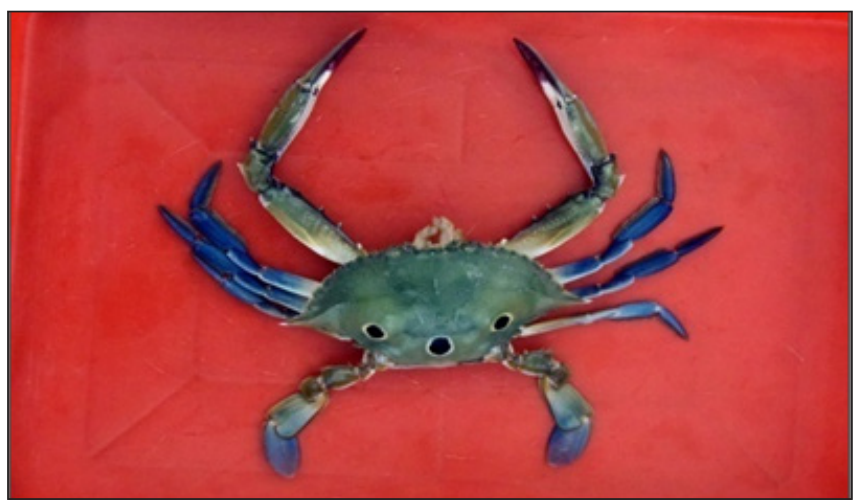

Portunus sanguinolentus

Figure 6: Shellfish species available at Kharakuva Fish market (Gujarat)

The ichthyofaunal diversity of Digha coast, West Bengal contributed 238 species from 72 families (Goswami, 1992), another study from similar region reported 212 species from 145 genera and 88 families (Chatterjee et al., 2000), further the most recent study from similar coast reported 322 fish species under 103 families (Yennawar et al., 2015). The study along the Bahuda estury, Orissa reported that there were 25 species under 6 orders and 18 families (Behera et al., 2013) were identified. Study also revealed that Rastrelliger kanagurta was dominant among the species (13.01\%), while order wise Clupiformes (30.08\%) was dominant which was followed by Mugiliformes, Tetradontiformes, Perciformes, Siluriformes and Cypridontiformes.

The ichthyofaunal diversity from two creeks of Port Blair was recorded a total of 1,701 individuals constituting 8 orders, 30 families and 42 genera (Arun Kumar et al., 2016). The study along the Great Nicobar Island from Bay of Bengal was reported a total of 258 species of fin fishes belonging to 141 genera, 84 families and 19 orders. Among these, order Perciformes represents 47 families, 84 genera and 169 species followed by Clupeiformes, Angulliformes, Tetrodoniformes, Cyprinodontiformes, Scorpaeniformes, Rajiformes, Elopiformes, Pleuronectiformes, Syluriformes, Laminiformes, Bercyformes, Aelopiformes, Syngathiformes and Gonorhychiformes. Study also revealed that the species which were reported are medicinally important and ornamentally valuable species (Rajaram et al., 2004). Another study from all the available habitats of the fresh water and marine environment of the Andaman and Nicobar Islands have reported 539 fish species (Rao et al., 2000).

The study from the sea and coastal waters of Gujarat, there were about 306 fish species were reported. Study also stated that the Gujarat fishery presently dominated by fishes like ribbonfishes (Trchiurus lepturus), Bombay duck (Harpodon nehereus), croakers, carangids, threadfin breams, lizardfishes, tuna (Euthynnus affinis, Thunnus tonggol, Katsuwonus pelamis, Thunnus albacores and Sarda orientalis), seerfish, pomfrets, catfish, flatfishes and non-penaeid prawns (Joshi et al., 2017). Another study from Gujarat waters about the ichthyofaunal diversity in the vicinity of marine protected areas, Jamnagar enumerated there were total of 109 fish species belongs to 19 orders, 58 families and 93 genera. Study also stated that Carangidae and Sciaenidae were two families recorded highest number of species diversity (8 species) which was followed by Mugilidae (5 spp.), Clupeidae (4 spp.), Haemulidae (4 spp.), Serranidae (4 spp.) and Sparidae (4 spp.; Brahmane et al., 2014). The similar study particular by considering the $\mathrm{Dol}$ net fishing was done along the Navabandar coast Gujarat (Sikotaria et al., 2018), study revealed that altogether 24 major fish and shellfish species were caught with Non-penaeid shrimps contributed $40.22 \%$ of the total landings, followed by Harpadon nehereus (Bombay duck; 15\%). The foreign water studies reported that along the inner Gulf of Nicoya, Pacific coast of Costa Rica, Central America there were total 274 species under 72 families were reported. The study further detailed as among all these species, 127 (46.4\%) were marine species and 147 (53.6\%) were estuarine-associated species (Murase et al., 2014).

\section{Conclusion}

The present landing center (Kharakuva, Veraval) from Saurashtra region of Gujarat represents the region is still under controlled fishing pressure. But during observations it was also reported that juveniles fishing was also started to contributing the landings. As fishes are immense important to the ecosystems, so they have to conserve, valued and 
managed properly. The study also describes the market value of the fishes, stated fish forms the economic as well as social importance to the Indian society.

\section{Acknowledgement}

The authors are grateful to College of Fisheries Science, Junagadh Agricultural University, Veraval for their support, encouragement and suggestions during the investigation during this study.

\section{Reference}

Anonymous, 2018a. FAO Catalogues. Available from http:// www.fao.org/fishery/org/fishfinder/3,3/en.

Anonymous, 2018b. Fish Base. Available fromhttp://fishbase. org.

Anonymous, 2018c. ITIS (Integrated Taxonomic Information System) Standard Report. Available from http://www. itis.gov.

Arun Kumar, M., Venu, S., Padmavati, G., 2016. Habitat Ecology and Ichthyofaunal Diversity of Two Creeks and Their Associated Streams from Port Blair, South Andaman Islands. International Journal of Ecology, 1-8. DOI: 10.1155/2016/1649368

Ayyappan, S., Moza, U., Gopalakrishnan, A., Meenakumari, B., Jena, J.K., Pandey, A.K., 2011. Handbook of Fisheries and Aquaculture. Indian Council of Agricultural Research, New Delhi, India.

Behera, D.P., Nayak, L., Sharma, S.D., 2013. A checklist on Ichthyofaunal diversity of Bahuda Estury, Odisha, East coast of India. International Journal of Zoological Research 9, 39-48. DOI: 10.3923/ijzr.2013.39.48.

Bene, C., Macfadyen, G., Allison, E.H., 2007. Increasing the Contribution of Small-scale Fisheries to Poverty Alleviation and Food Security. Food and Agriculture Organization of the United Nations, Rome.

Bijukumar, A., Sushama, S., 2000. Ichthyofauna of Ponnani estuary, Kerala. Journal of Marine Biological Association of India 42, 182-189.

Brahmane, V.T., Temkar, G.S., Metar, S.Y., Sikotaria, K.M., Desai, A.Y., 2014. Ichthyofaunal diversity in vicinity of marine protected areas, Jamnagar, Gulf of Kachchh, India. Asian Journal of Advanced Basic Sciences 3, 78-88.

Chatterjee, T.K., Ramakrishna, Talukdar, S., Mukherjee, A.K., 2000. Fish and Fisheries of Digha coast of West Bengal. Rec. Zool. Surv. India. Occ. Paper 188, 1-74.

CMFRI (Central Marine Fisheries Research Institute), 2017. Annual Report 2016-17. Central Marine Fisheries Research Institute, Kochi, 292.

Day, F., 1878. Fishes of India. Willium Dawson's, London U. K. reprint edition, Today and Tomorrow Book agency, Delhi.

Dulvy, N.K., Metcalfe, J.D., Glanville, J., Pawson, M.G., Reynolds, J.D., 2000. Fishery stability, local extinctions and shifts in community structure in skates. Conserv. Biol. 14, 283-293.
Geoffrey, P., Jone, S., Srinivasan, M., Glenn, R.A., 2007. Population Connectivity and Conservation of Marine Biodiversity. Oceanography 20, 100-111.

Goswami, B.C.B., 1992. Marine fauna of Digha Coast of West Bengal, India. Journal of Marine Biological Association of India 34, 115-137.

Hilborn, R., Quinn, T.P., Schindler, D.E., Rogers, D.E., 2003. Biocomplexity and Fisheries Sustainability. Ecol. Monogr. 75, 3-3.6.

IUCN (International Union for Conservation of Nature), 2008. Status of the World's Marine Species.The IUCN Red List of Threatened Species, Species Survival Commission, 1-2.

Johnson, D.W., Freiwald, J., Bernardi, G., 2016. Genetic diversity affects the strength of population regulation in a marine fish. Ecology 97, 627-639.

Joshi, K.K., Thobias, P.A., Varsha, M.S., 2017. Present status of ichthyofaunal diversity of Indian seas. In: Course Manual Summer School on Advanced Methods of Fish Stock Assessment and Fisheries Management. Lecture Note Series No. 2/2017. Central Marine Fisheries Research Institute (CMFRI), Kochi, Kerala, 1-22.

Kabat, P., Bazelmans, I., Dijk, J.V., Herman, P.M.J., Oijen, T.V., Pejrup, M., Reise, K., Speelman, H., Wolff, W.J., 2012. The Wadden Sea Region: Towards a science for sustainable development. Ocean \& Coastal Management 68, 4-17. DOI: 10.1016/j.ocecoaman.2012.05.022

Kar, D., 2003. Fishes of Barak drainage, Mizoram and Tripura. In: Kumar, A., Bohra, C., Singh, L.K. (Eds.),Environment, Pollution and Management. APH Publishing Corporation, New Delhi, 203-211.

Kumaran, B., Kambala, N.S., Nadarajan, J., 2012. Assessment of Ichthyofaunal diversity in Giriyampeta Estury, Yanam (U.T. of Puducherry). Bulletin of Environment, Pharmacology and Life Sciences 1, 17-25.

Levque, C., Oberdorff, T., Paugy, D., Satiassny, M.L.J., Tedsco, P.A., 2008. Global diversity of Fish (Pisces) in Freshwater. Hydrobiologia 595, 545-567. DOI: 10.1007/s10750-0079034-0

Murase, A., Angulo, A., Miyazaki, Y., Bussing, W.A., López, M.I., 2014. Marine and Estuarine Fish Diversity in the Inner Gulf of Nicoya, Pacific Coast of Costa Rica, Central America. Check List 10, 1401-1413. DOI: 10.15560/10.6.1401

Rahbek, C., Colwell, R.K., 2011. Biodiversity: Species loss revisited. Nature 573, 288-289.

Rajaram, R., Srinivasan, M., Khan, S.A., Kannan, L., 2004. Ichthyofaunal Diversity of Great Nicobar Island, Bay of Bengal. Journal of the Indian Fisheries Association 31, 13-26.

Rajasegar, R., Sendhilkumar, R., 2009. Finfish Resources of Karaikal, South East Coast of India. World Journal of Fish and Marine Sciences 1, 330-332. DOI: 10.1.1.608.6600

Rao, D.V., Kamala, D., Rajan, P.T., 2000. An account of Ichthyofauna of Andaman and Nicobar Islands, Bay of 
Bengal. Records of the Zoological Survey of India Occ. Paper No. 178, 1-434.

Sikotaria, K.M., Temkar, G.S., Azeez, P.A., Mathew, K.L., 2018. Ichthyofaunal Diversity of Dol net Fishery at Navabandar, Gujarat. International Journal of Bio-resource and Stress Management 9, 108-113. DOI: 10.23910/ IJBSM/2018.9.1.3C0599

Talwar, P.K., 1991. Pisces, Animal resources of India: Protozoa to Mammalia. Zoological Survey of India Calcutta 1, 577-630.

Talwar, P.K., Jhingran, A.G., 1991. Inland Fishes of India and Adjacent countries. Oxford \& IBH Co. Pvt. Ltd, New Delhi.

Venkataraman, K., 2006. Biodiversity Legislations in Like Minded Megadiverse Countries.In: Verma, D.D., Arora, S., Rai, R.K. (Eds.), Prospectives on Biodiversity: A vision for Megadiverse Countries.Ministry of Environment and Science, Center for Environment Education, Ahmedabad, 79-92.

Walters, C., Pearse, P.H., 1996. Stock Information Requirements for Quota Management Systems in Commercial Fisheries. Reviews in Fish Biology and Fisheries 6, 21-42.

William, N.E., Ronald, F., Jon, D.F., Dennis, A.P., 2010. Marine Fish Diversity: History of Knowledge and Discovery (Pisces). Zootaxa 2525, 19-50.

Yennawar, P., Mohapatra, A., Ray, D., Tudu, P., 2015. Ichthyofauna of Digha Coast, India. Marine Faunal Diversity in India 1, 235-248. DOI: 10.1016/B978-0-12801948-1.00015-X. 